\author{
Regrinted from IEEE TRANSACTIONS \\ ON EL.RCTRON DEVICES \\ Volume ED-16, Number 8, August, 1960 \\ pp. $72,-728$

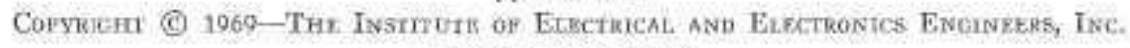 \\ MTNTEO LN THE D, D.A.
}

\title{
Effect of Cesium Pressure on Thermionic Stability
}

\author{
ALFRED SCHOCK
}

\begin{abstract}
It is shown that under certain conditions of heat input, reservoir temperature, and load voltage or resistance a thermionic converter can equilibrate at two radically different operation points, corresponding to conditions of high and low cesium coverage. Moreover, abrupt transitions between these operating regimes, accompanied by a temperature rise of hundreds of degrees, can occur whenever the critical heat generation rate for a given reservoir temperature is exceeded. To provide an adequate safety margin against such an occurrence, thermionic systems must be operated at relatively high cesium pressures, even though this may cause some performance degradation. This paper consists of two parts. The first explains the above effect with reference to a single converter. The second part illustrates the effect of cesium reservoir temperatures on the dynamic behavior of an open-loop thermionic reactor following a small reactivity perturbation.
\end{abstract}

\section{INTRODUCTION}

I IS customary for thermionic system designers to choose the optimum operating point by maximizing some figure of merit, such as power density, effciency, or power per unit weight. The present paper seeks to demonstrate that the choice of operating parameters, particularly the cesium reservoir temperature, must also take account of a factor we shall refer to as "thermionic stability". In fact, under certain conditions this factor must dominate over other considerations in

Manuscript received December 9, 1968; revised March 31, 1969. This work was supported by the U. S. Atomic Energy Commissiont. This paper was ptesented at the IEEE Thermionic Specialists Conference, Framingham, Mass, October 21-23, 1968.

The author is with Republic Aviation Division, Fairchild Hiller Corporation, Fartiningdale, N. Y. order to achieve a stable system and avoid excessive temperature excursions.

In practical applications, thermionic converters typically operate under conditions of constant voltage or constant load resistance. Under these conditions relatively small perturbations of load or heat input can lead to extensive desorption of cesium, with a consequent large rise in emitter temperature. We designate this phenomenon as "thermionic burnout" because of its similarity to burnout in boiling heat transfer. This condition had been postulated by the author in an carlier paper [1] on the basis of purely qualitative arguments. The recent development of the simcon code [2], [3], for the theoretical extrapolation of thermionic performance data over a very wide range of temperatures has made it possible to demonstrate this effect on a quantitative basis. Recent work [4] has provided experimental verification of the predicted thermal instability effect.

\section{EFFEcT OF EMTTTER ThMperature on Current Density}

To clarify the subsequent discussion, let us first examine typical curves of current density versus emitter temperature, as illustrated by Fig. 1. All data are for a tungsten emitter (with a $4.75-\mathrm{eV}$ bare work function) and a $100^{\circ} \mathrm{K}$-molybdenum collector, separated by a $0.025-\mathrm{cm}$ gap. The bottom curve represents the saturated emission of the bare tungsten emitter. The other curves are for a fixed converter potential of 0.6 volt, and 


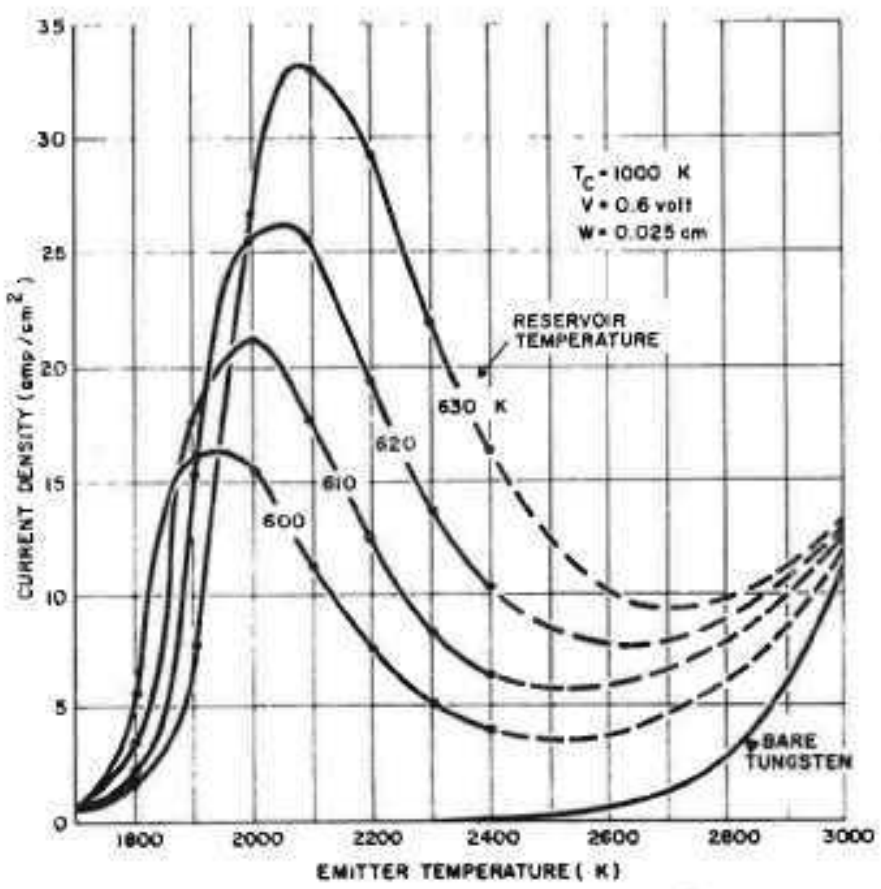

Fig. 1. Effect of temperature on current density.

represent constant cesium reservoir temperatures of $600,610,620$, and $630^{\circ} \mathrm{K}$, respectively.

For emitter temperatures up to $2400^{\circ} \mathrm{K}$, the curves were produced by a special "thermionic correlation" (TICORR) computer code. The program operates on a large array of simcon-generated [2] current-voltageheat flux data, covering a wide range of emitter temperatures $\left(1700-2400^{\circ} \mathrm{K}\right)$, collector temperatures ( $850-$ $\left.1150^{\circ} \mathrm{K}\right)$, and reservoir temperatures $\left(550-690^{\circ} \mathrm{K}\right)$. TICORR performs multidimensional interpolations, and automatically produces a wide variety of cross-plots, not only of the above six parameters but also of power density, efficiency, and specific load conductance.

Construction of the curves above $2400^{\circ} \mathrm{K}$ was based on the fact that each curve must asymptotically approach the bare emitter curve at sufficiently high temperatures. Since the subsequent discussion is primarily concerned with the qualitative nature of the effect, it is sufficient to draw smooth transitions between the TICORR plots and the bare emitter curve; as illustrated by the dashed curves in Fig. 1.

As can be seen, each of the constant cesium pressure curves passes through a maximum and a minimum. The direct effect of raising the emitter temperature is to increase the current density, while its indirect effect is to reduce the cesium coverage, raise the emitter work function, and thereby lower the current density. At temperatures below the current maximum and above the current minimum the direct effect predominates, while at intermediate temperatures the indirect effect predominates. This is similar to the Langmuir S-curves [5] except that we are dealing with constant-voltage diode currents rather than saturated emission.

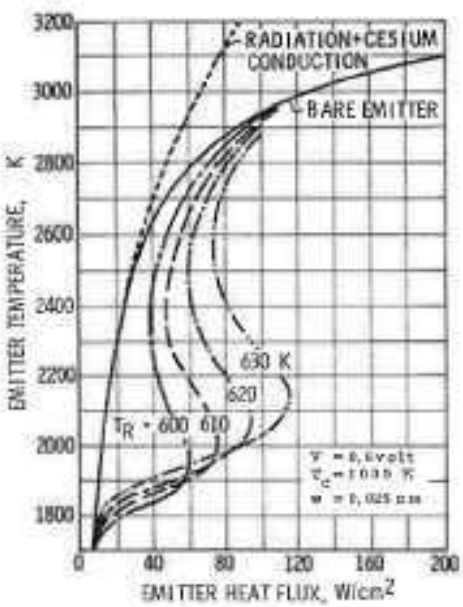

Fig. 2. Variation of temperature with heat flux.

\section{EMITIER HEAT FLUX}

Fig. 2 presents a plot of emitter temperature versus heat flux corresponding to the data shown in Fig. 1. The leit-most curve represents the emitter heat flux due to radiation and cesium conduction. (Actually, cesium conduction varies with reservoir temperature, but this variation is generally small compared with the total heat flux.) The curve marked "bare emitter" contains the additional heat flux due to saturation-current electron cooling from the uncesiated tungsten surface. As can be seen, at very high temperatures this makes a quite appreciable contribution.

Finally, the constant cesium pressure curves contain the electron cooling heat flux corresponding to the current densities depicted in Fig. 1. Each curve again 
passes through a maximum and a minimum. As would be expected, the temperature at which the maximum heat flux occurs increases with cesium pressure, as does the magnitude of the maximum heat flux.

\section{OPEN-CIRCUIT EFFECT}

Before proceeding to the principal subject of this paper, it should be noted that Fig. 2 provides a very graphic illustration of the open-circuit temperature rise, which results from loss of electron cooling. The curves reveal that there is a rather substantial difference between a literal open-circuit, i.e, a complete break in the diode-to-load circuit, and the much more likely nominal "open-circuit", i.e., loss of cesium due to a small leak (as long as sufficient cesium remains for space charge neutralization, $\sim 10^{-5}$ torr $\rangle$. This can be seen by comparing the bare-emitter and the radiation and conduction curves in Fig. 2.

\section{BistaBle CONVBRTER Operation}

To appreciate the significance of the curve shapes shown in Fig. 2, let us examine at single such curve, as illustrated in Fig. 3. Only this time the abscissa should be interpreted not as the emitter heat flux, but as the heat generation rate per unit emitter area. As can be seen, for any heat generation rate between points $A$ and $D$ the various simcon equations $[2]$ are sa tisfied at three distinct emitter temperatures, corresponding to high, medium, and low cesium coverage. However, only two of these represent stable operating conditions, since the middle point will be shown to be inherently unstable. At which of the two outer points the converter operates must depend on its previous history.

\section{CONVERTER HYSTEResis}

Consider a converter starting from equilibrium at point 1 , whose heat generation rate is increased in a series of small steps, with sufficient time intervals between steps to allow the system to equilibrate. Each increase in heat generation produces a small increase in emitter temperature, until point $D$ (the cesium desorption point is approached. Any further increase in heat generation, however amall, leads to a condition in which the generated heat exceeds the heat leaving the emitter, resulting in a steady temperature rise. $\mathrm{As}$ the emitter temperature rises, its heat loss rate (which follows the dashed portion of the curve) diminishea. This further increases the lack of equilibrium, causing the temperature rise to accelerate. Clearly, the temperature will continue to go up until point 2 on the low-cesiumcoverage branch of the curve is reached, where equilibrium is reestablished. Any further increase in heat generation will cause the operating point to follow the upper branch in a normal fashion.

Conversely, when the heat generation rate is decreased from point 2, the operating point will follow

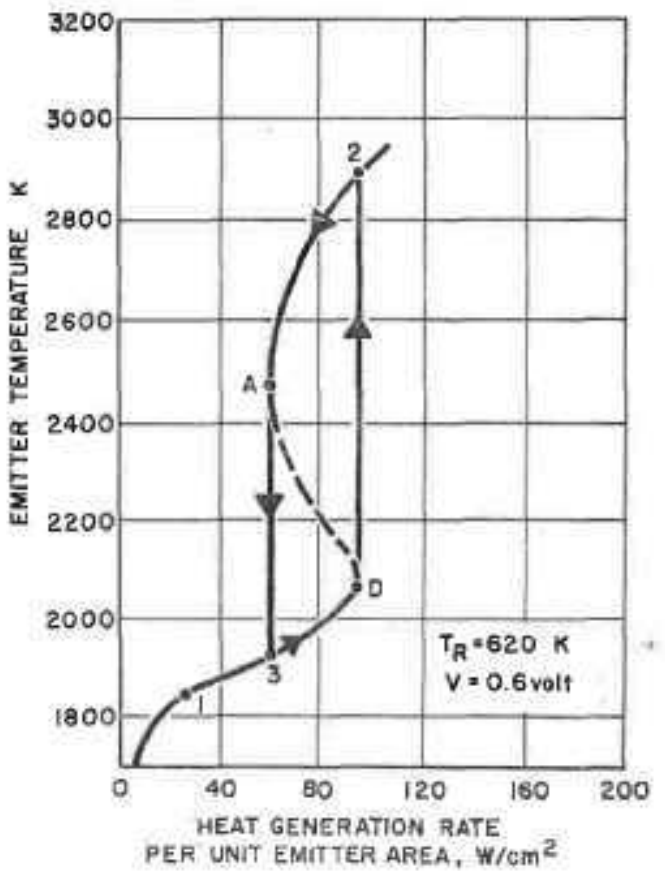

Fig. 3. Efiect of varying heat generation rate.

the upper branch until point $A$ (the cesium adsorption point) is reached. Any further decrease in heat generation will cause an abrupt drop in temperature, until equilibrium is reestablished at point 3 on the highcesium-coverage branch of the curve. This, the overall cycle is seen to exhibit a hysteresis effect, completely. bypassing the middle (negative slope) branch of the curve,

Although it might be possible, by some transient maneuver, to bring the converter to equilibrium at some point on the middle branch of the curve, such equilibrium would be inherently unstable, since even a slight perturbation of the heat generation rate (or load resistance) would drive the converter to either the upper or lower branch of the curve.

\section{Burnout Margin}

The principal significance of the phenomena discussed above is that they introduce an additional constraint in choosing the converter operating point. For example, with a maximum emitter temperature of $2000^{\circ} \mathrm{K}$ and a 0.6 -volt load, Fig. 2 shows that it is not practical to employ reservoir temperatures at or below $610^{\circ} \mathrm{K}$, even though such temperatures may yield the highest conversion efficiencies.

Even a reservoir temperature of $620^{\circ} \mathrm{K}$ would provide scant margin for safety, since the diode operating point could be shifted to the high temperature $\left(3000^{\circ} \mathrm{K}\right)$ branch of the curve by a minor increase in heat generation rate (e.g., from 87 to $\left.93 \mathrm{~W} / \mathrm{cm}^{\circ}\right)$. Such small deviations, either spatial or temporal, would be very difficult to avoid in a thermionic reactor. It therefore appears that to operate at $2000^{\circ} \mathrm{K}$ with a fixed 0.6-volt load, one 


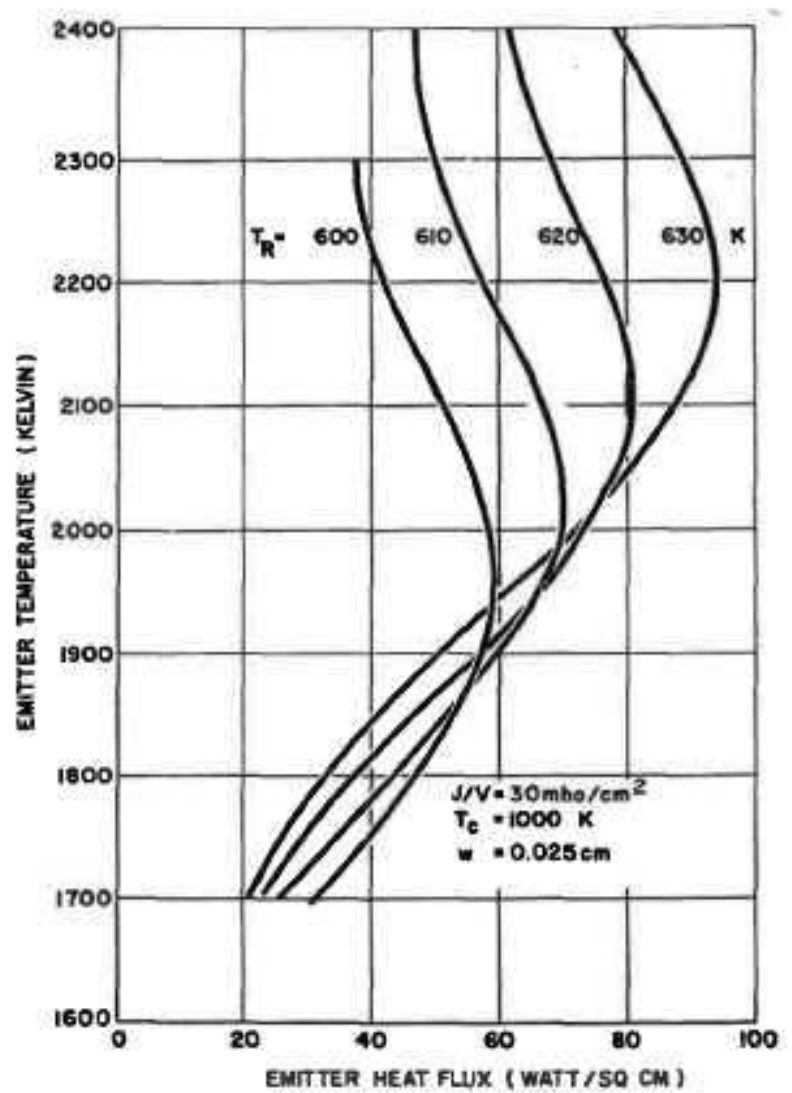

Fig, 4. T-q variation at constant load resistance.

should employ a reservoir temperature of $630^{\circ} \mathrm{K}$ or more. In that case the burnout condition, i.e., the cesium desorption point, is not reached until the heat generation rate increases from 89 to $116 \mathrm{~W} / \mathrm{cm}^{2}$, providing a comfortable 30 percent safety margin.

The precise numbers in the above discussion would vary somewhat for different load voltages, or for a fixedresistance rather than fixed-voltage load. However, the general principles discussed above would still apply. This is illustrated by Fig. 4 for a constant load conductance of 30 mho per $\mathrm{cm}^{2}$ of emitter.

\section{REACTOR DYNAMiCS}

Finally, it is of interest to examine the dynamic behavior of a thermionic reactor passing through the cesium desorption point. To do so, we make use of a specialized digital transient analysis code developed at Republic Aviation. The code combines a zero-dimensional nuclear analysis using six delayed neutron groups with a 2-dimensional $(r z)$ thermal analysis of a single thermionic fuel element. The program uses the TICoRR code to compute the instantaneous emitter heat flux. It permits step changes in reactivity, separate fuel and emitter temperature feedback coefficients, and ramp insertion of scram rods with programmed time delay.

The code automatically produces plots of heat generation and heat loss rates per unit emitter area; also of reactivity, emitter temperature, and maximum fuel temperature. Such computer-produced plots are shown in Figs, 5 and 6 . Both figures portray the behavior of an open-loop reactor following a 2 -cent reactivity step change, and are based on the identical converter geometry (the details of which are not important for the present discussion). The reactivity feedback coefficients were taken to be $+0.001 \mathrm{k} /{ }^{\circ} \mathrm{K}$ for the fuel and $-0.020 \mathrm{k} /{ }^{\circ}$ $\mathrm{K}$ for the emitter, with a $1-\mu \mathrm{s}$ prompt neutron lifetime. Both figures assume a constant collector temperature of $1000^{\circ} \mathrm{K}$, and initial equilibrium at an emitter temperature of $2000^{\circ} \mathrm{K}$. (The difference between the heat generation and loss rates during the transient has been shaded in both figures, to bring out the net heat surplus or deficit.)

\section{EFGECT OF RESERVOR TEMPRRATURE on Reactor Dynamics}

The principal difference between the figures is the postulated cesium reservoir temperature, which is $630^{\circ} \mathrm{K}$ in Fig. 5 and $620^{\circ} \mathrm{K}$ in Fig. 6. In the former case, it is seen that, as the result of the 2 -cent reactivity step, the heat gain and loss rates slowly rise from 88 to 113 $\mathrm{W} / \mathrm{cm}^{2}$, where a new thermal and nuclear equilibrium is established. During this process, which occurs over a period of several minutes, the emitter temperature rises by approximately $95^{\circ} \mathrm{K}$, without any temperature overshoot. Note that the new emitter temperature is still below the cesium desorption point for a $630^{\circ} \mathrm{K}$ reservoir.

By contrast, consider the effect of the same reactivity perturbation in the case of a $620^{\circ} \mathrm{K}$ reservoir temperature, shown in Fig. 6. As before, the stepped up reactivity produces a prompt jump in heat generation; this causes a gradual rise in temperature, which in turn lowers the reactivity and raises the heat loss rate. However, after 1.5 minutes the cesium desorption point $D$ is passed, and the subsequent loss in cesium coverage causes a sharp drop in the emitter cooling rate. This continues until point $A$ is passed at $t=3.4$ minutes, when the heat loss starts to rise again.

Meanwhile, because of the large heat surplus, the emitter and fuel temperatures have risen by hundreds of degrees. As a result of the emitter feedback coefficient, the reactivity drops sharply passing through the criticality point at $t=1.9$ minutes, leading to a decrease in the heat generation rate. Eventually, at $t=3.7$ minutes, the heat gain and loss rates cross, the emitter and maximum fuel temperatures peak at 2534 and $2650^{\circ} \mathrm{K}$, respectively, and the reactivity bottoms at -7.98 cents.

The subsequent heat deficit causes the temperature to diminish slowly until, at $t=4.1$ minutes, the cesium adsorption point $A$ is passed again. After this, the increased cesium coverage produces a sharp increase in the heat loss rate with a corresponding drop in temperature. 


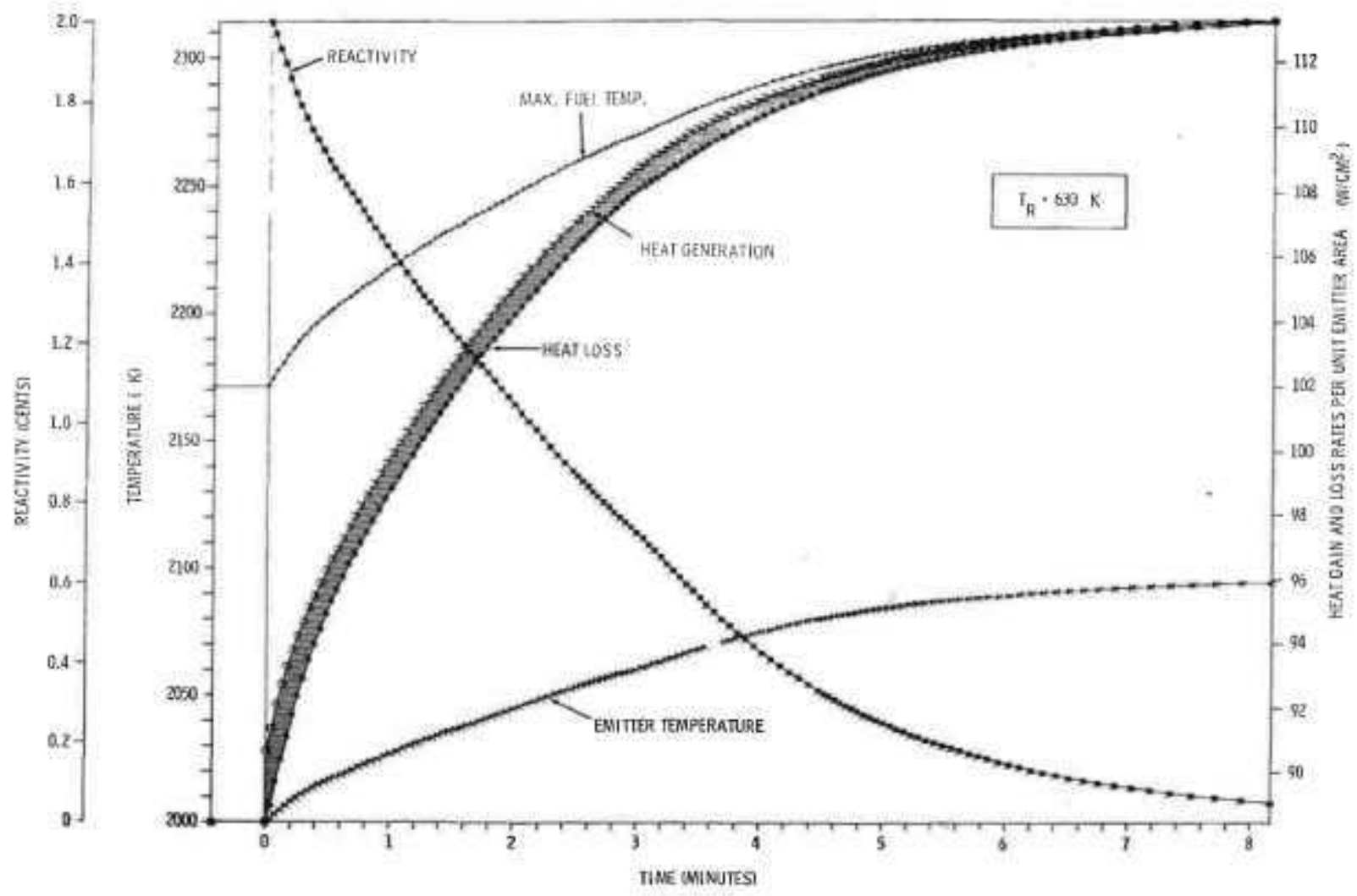

Fig. 5. Effect of 2 -cent step with $630^{\circ} \mathrm{K}$ reservoir.

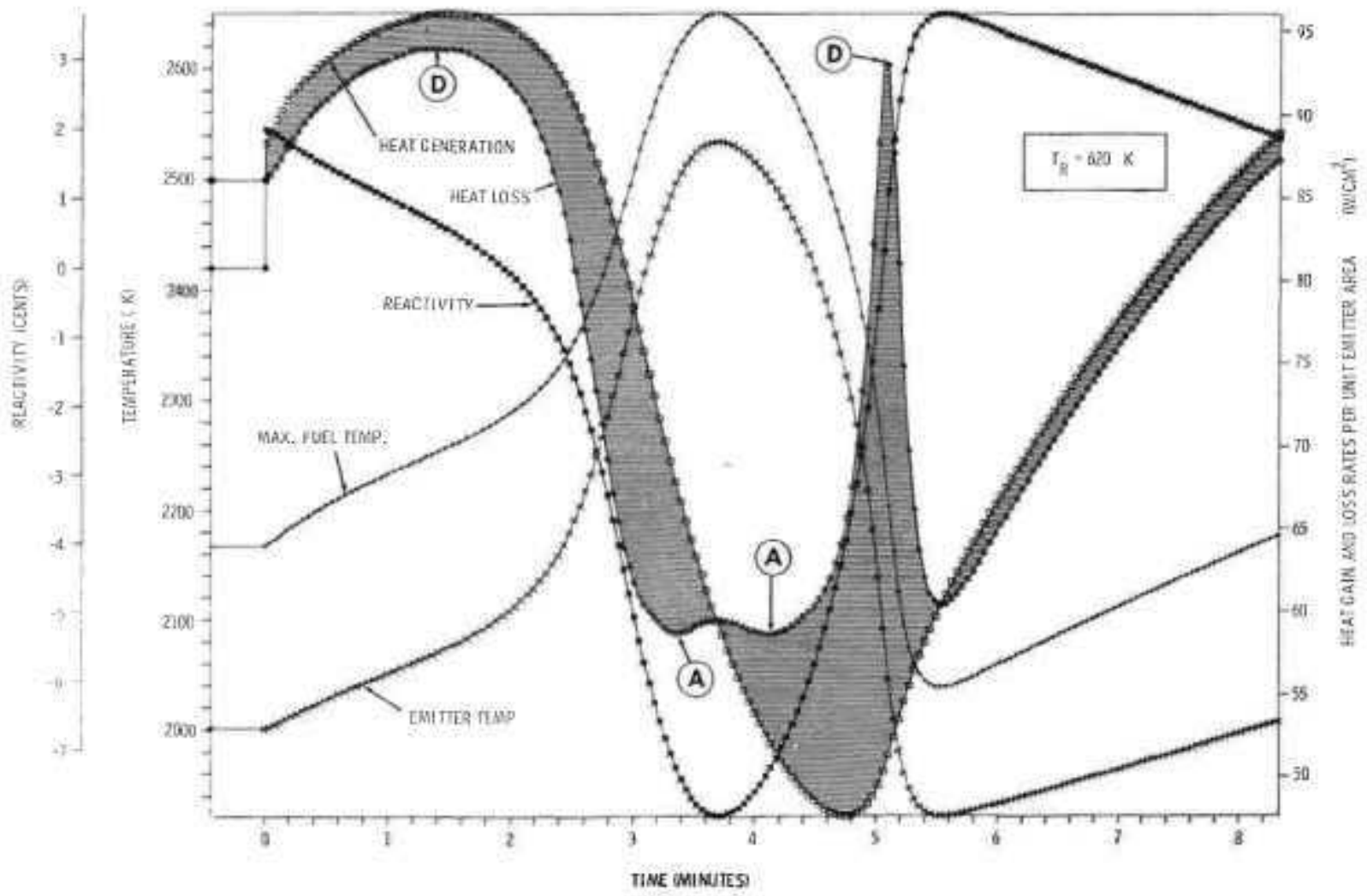

Fig. 6. Effect of 2-cent step with $620^{\circ} \mathrm{K}$ reservoir. 
At $t=5.1$ minutes point $D$ is passed, and the heat loss rate drops sharply until it crosses the rising heat production curve at $t=5.5$ minutes, at which point the emitter tenperature reaches a low point of $1920^{\circ} \mathrm{K}$ and the reactivity peaks at +3.5 cents. Although Fig. 6 is cut off at 8 minutes, extended computations have shown that the cycle essentially repeats indefinitely. Within the assumptions of the particular problem, there is no damping mechanism and the system would never reach equilibrium.

\section{Conctustons}

In a sense, the results presented are somewhat reassuring, since they show that the various changes occur quite slowly, allowing ample time for the control system to react. This relative sluggishness arises from the high heat capacity of the fuel-emitter assembly. It may therefore be argued that the problem is not really of practical interest, since the type of transients described above would be prevented by the control system. The argument is flawed for two reasons. Even if it is feasible to do so, it is not very desirable to operate a basically unstable system, where the control system must be constantly active to prevent small perturbations from causing large temperature excursions.

Moreover, even if it were practical to avoid the above type of transients for the reactor as a whole, this still would leave the problem of undetected burn-out in individual converters. That would be a danger if the design operating point were close to the cesium desorption point, since some variation among converters is unavoidable. One way of alleviating the changes would be to employ integral cesium liquid or adsorption reservoirs thermally coupled to the emitter. Thus, burn-out could be avoided in principle if an increase in emitter temperature automatically led to an appropriate rise in cesium pressure. For the general case of a thermally uncoupled reservoir, the transient studies presented above confirm the desirability of operating at a higher cesium pressure, i.e, $630^{\circ} \mathrm{K}$ in the present instance, to provide a reasonable margin of safety.

\section{ACKNOWLEDGMENT}

The author wishes to express his thanks to C. L. Eisen for his valuable help, particularly in the construction of the transient analysis program, and to Dr. D. R. Wilkins for furnishing the tables of stscos-gentrated thermionic performance data.

\section{REFERENCES}

[1] A. Schock, "Effect of non-uniform heat generution on thermionic reactors," Proc. IEEE Thermionic Conversion Specialist Conf. (Houston, Tex. Noyember 1966).

[2] D. R. Witkins, "An improved theoretical description of thermionic converter performance characteristics," Rept. $27 \mathrm{th}$. Amm. Cowf. on Plyssical Electronics (Massachusetts Institute of Technology, Cambridge, Mass, March 1967).

[3] D. R. Wilkins, C. D. Sawyer, and P. R. Hill, "Theory of thermionic conversion operation with applications to thermiontic reactor analysis," Second Ententat. Conf, on Thermionic Electrical Poner Generation (Stresa, Italy, May 1968), pp. 799-812

[4] D. Lieb and F. Rufeh, Thermal seability as a function of converter performance," Proc. IEEE Thermionic Comsersion Spccialist Conf. (Framingham, Mass., October 1068), pp, 318-322.

[5] V. C. Wilson, in Direct Comersion of Heal to Electricity, J. Kaye and J. Welsh, Eds. New York: Wiley, 1960, pp. 7,1 to $7-12$. 